\section{Modification of $\mathrm{KCNH} 2$-Encoded Cardiac Potassium Channels by KCNE1 Polymorphism}

\section{To the Editor:}

We read with great interest the recent study by Dr Yamaguchi and colleagues in which they reported modulatory effects of the glycine/serine polymorphism (G38S) of KCNE1 on ionic current carried by KCNQ1 (Kv7.1) and $K C N H 2$ (Kv11.1 or "hERG") potassium channels. ${ }^{1} \mathrm{KCNE} 1$ is perhaps best known as the $\beta$ subunit of potassium channels carrying the cardiac slow delayed rectifier current IKs (of which KCNQ1 forms the $\alpha$ subunit), but it can also interact with hERG (the major subunit of channels carrying the rapid delayed rectifier potassium current, IKr). As both IKr and IKs are involved in ventricular repolarization, mutations or polymorphisms that reduce these currents can either lead to or predispose to long QT syndrome (LQTS). ${ }^{2}$ hERG is also particularly susceptible to pharmacological blockade and so is strongly implicated in the drug-induced form of LQTS (diLQTS). ${ }^{3}$ diLQTS is multifactorial; frequently, other risk factors in addition to the presence of a drug that inhibits hERG are present in cases of torsades de pointes. ${ }^{3}$ The identification of additional factors that may exacerbate drug block of hERG is therefore of some importance.

In their recent paper, Yamaguchi et al have shown differences between the major (glycine) and minor (serine) polymorphisms at residue 38 of KCNE1 in terms of their effects on KCNQ1 and on hERG. KCNE1-38S produced a positive shift in voltage-dependent activation of KCNQ1+KCNE1, compared to KCNQ1+KCNE1-38G. ${ }^{1}$ Of particular note, however, are the differences reported between the 2 polymorphisms when coexpressed with hERG. With a conventional voltage-clamp protocol, hERG current magnitude was significantly smaller for hERG+KCNE1-38S than with KCNE1-38G at potentials of $-20 \mathrm{mV}$ and more positive, without any accompanying shift in the voltage-dependence of activation. However, when hERG was co-expressed with both KCNE1 polymorphisms (to mimic the heterozygous state), current magnitude was not significantly depressed compared to that for hERG+KCNE1-38G. The authors also performed some pharmacological experiments, reporting that the sensitivity of hERG to the selective hERG/IKr inhibitor E-4031 was greater for hERG+KCNE1-38S than for hERG+KCNE1-38G (the IC50 for the latter being 4.2-fold that of the former). ${ }^{1}$ hERG co-expression with the minor $38 \mathrm{~S}$ KCNE1 polymorphism increased the sensitivity of reactivation (recovery from inactivation) kinetics to hypokalemia. ${ }^{1}$ The hERG results in their study are consistent with a modest depression of $\mathrm{IKr}$ in a setting of homozygous KCNE1-38S and, potentially, to differential effects of IKr blockade between the major and minor KCNE1 polymorphisms studied.

Effects of mutations/polymorphisms in KCNE1 in terms of susceptibility to diLQTS are most readily considered in relation to effects on IKs and consequent reduction of repolarization reserve. The results of this new study ${ }^{1}$ highlight that consideration should not necessarily be limited to IKs; additional potential contributory factors are a reduction in IKr amplitude and increased sensitivity of IKr to pharmacological blockade. E-4031 is an experimental rather than clinically used drug, but is struc- turally related to agents (dofetilide, sotalol; all 3 being methanesulfonanilides) that are. It would be interesting to determine whether clinically used methanesulfonanilides also exhibit differential actions between KCNE1-38G and KCNE1-38S. The pharmacology was also conducted at room temperature rather than $37^{\circ} \mathrm{C}$ and complementary pharmacological data at physiological temperature would be useful. Nevertheless, we believe that these findings are potentially highly significant when considered alongside results of a recent study of ours in which the effects of 3 naturally occurring KCNE1 variants (A8V, D76N and $\mathrm{D} 85 \mathrm{~N}$ ) on hERG current and pharmacology were investigated at physiological temperature. ${ }^{4}$ Using both conventional voltage clamp and ventricular action potential voltage clamp, we found the magnitude of the hERG current to be markedly suppressed by each KCNE1 variant compared to wild-type (WT) KCNE1. Moreover, the $3 \mathrm{KCNE} 1$ variants that we studied influenced the sensitivity of hERG to pharmacological blockade by 2 of 3 clinically used drugs that we investigated. ${ }^{4}$ Thus, although hERG sensitivity to the Class Ia antiarrhythmic, quinidine, was similar between WT KCNE1 and the 3 mutant variants studied, that for the antibiotic clarithromycin and for the gastric prokinetic drug, cisapride, differed between the KCNE1 variants studied. ${ }^{4}$ Our findings demonstrate that mutations to KCNE1 can influence hERG pharmacology for some but not all drugs. ${ }^{4}$ Consequently, our recent study and that of Yamaguchi et al $^{1}$ independently provide mutually supportive evidence consistent with a potentially significant modulatory role of KCNE1 variants on hERG in relation to diLQTS.

\section{Acknowledgments}

We thank Heart Research UK for funding (RG2594).

\section{Disclosures}

Conflicts of Interest: None for any authors.

\section{References}

1. Yamaguchi Y, Nishide K, Kato M, Hata Y, Mizumaki K, Kinoshita $\mathrm{K}$, et al. Glycine/serine polymorphism at position 38 influences KCNE1 subunit's modulatory actions on rapid and slow delayed rectifier $\mathrm{K}^{+}$ currents. Circ J 2014; 78: 610-618.

2. Modell SM, Lehmann MH. The long QT syndrome family of cardiac ion channelopathies: A HuGE review. Genet Med 2006; 8: 143-155.

3. Kannankeril PJ, Roden DM. Drug-induced long QT and torsade de pointes: Recent advances. Curr Opin Cardiol 2007; 22: 39-43.

4. Du C, El Harchi A, Zhang H, Hancox JC. Modification by KCNE1 variants of the hERG potassium channel response to premature stimulation and to pharmacological inhibition. Physiol Rep 2013; 1: e00175, doi:10.1002/phy2.175.

Jules C Hancox, $\mathrm{PhD}$

Chunyun Du, PhD

Aziza El Harchi, PhD

School of Physiology and Pharmacology and Cardiovascular Research Laboratories, Medical Sciences Building, University of Bristol, Bristol, UK Henggui Zhang, PhD

Biological Physics Group, School of Physics and Astronomy, University of Manchester, Manchester, UK

(Released online July 10, 2014) 